\title{
Up-regulation of Grb2-associated binder 1 promotes hepatocyte growth factor induced endothelial progenitor cell proliferation and migration
}

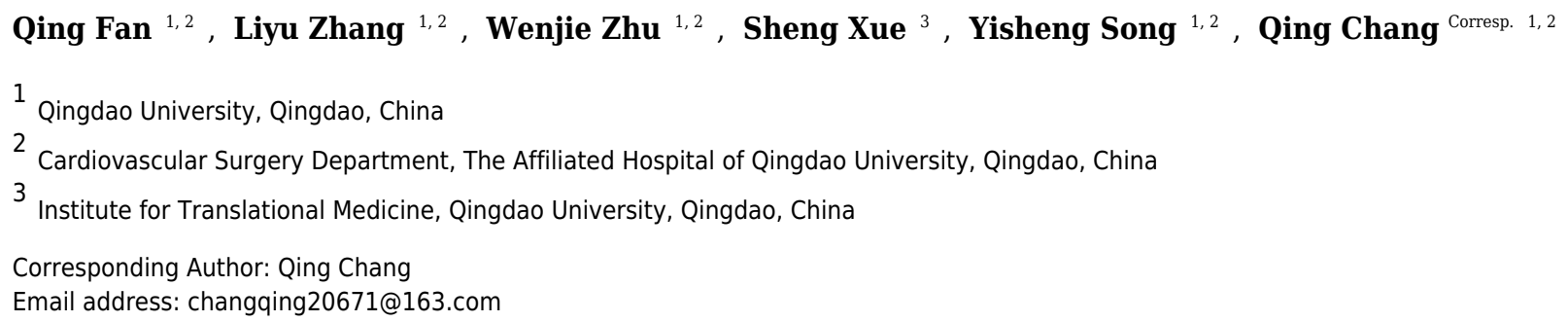

Objectives. Grb2-associated binder 1 (Gab1), a scaffolding adaptor protein, plays an important role in transmitting key signals that control cell growth, migration, and function from multiple tyrosine kinase receptors. This study was designed to investigate the influence of upregulation of Gab1 in endothelial progenitor cells (EPCs) stimulated with hepatocyte growth factor (HGF), and the underlying molecular mechanisms.

Materials and Methods. EPCs isolated from human umbilical cord blood were identified and divided into four groups. EPCs in the Control group were cultured normally; those in the Control+HGF group were treated with HGF stimulation; those in the AD-Gab1 group were transfected with adenovirus containing the Gabl gene but not treated with HGF stimulation; and, those in the AD-Gab1+HGF group were treated with both HGF stimulation and transfection with adenovirus containing the Gabl gene. Subsequently, Gab1 expression and proliferation and migration ability were compared for EPCs grown under different conditions. Furthermore, we measured phosphorylation levels of three key proteins Gab1, SHP2 and ERK1/2.

Results. The AD-Gab1+HGF group had the highest expression of Gab1 and higher proliferation and migration than the other three groups.

Conclusions. Upregulation of Gab1 promoted HGF-induced EPC proliferation and migration. Mechanistically, HGF stimulated Gabl tyrosine phosphorylation in EPCs, thus leading to activation of extracellular regulated MAP kinase $1 / 2$, which is involved in proliferation and migration signaling. 


\section{Up-regulation of Grb2-associated Binder 1 Promotes}

2 Hepatocyte Growth Factor Induced Endothelial

3 Progenitor Cell Proliferation and Migration

Qing Fan ${ }^{1,2}$, Liyu Zhang ${ }^{1,2}$, Wenjie Zhu ${ }^{1,2}$, Sheng Xue ${ }^{3}$, Yisheng Song ${ }^{1,2}$, Qing Chang ${ }^{2}$

$6 \quad{ }^{1}$ Qingdao University, Qingdao 266071, China

$7{ }^{2}$ Cardiovascular Surgery Department, The Affiliated Hospital of Qingdao University, Qingdao

8 266003, China

$9 \quad{ }^{3}$ Institute for Translational Medicine, Qingdao University, Qingdao 266021, China

10

11

12

Corresponding Author:

Qing Chang

Cardiovascular Surgery Department, The Affiliated Hospital of Qingdao University, Qingdao 266003, China

Email address: changqing20671@163.com

\section{Abstract}

Objectives. Grb2-associated binder 1 (Gab1), a scaffolding adaptor protein, plays an important role in transmitting key signals that control cell growth, migration, and function from multiple tyrosine kinase receptors. This study was designed to investigate the influence of upregulation of Gab1 in endothelial progenitor cells (EPCs) stimulated with hepatocyte growth factor (HGF), and the underlying molecular mechanisms.

Materials and Methods. EPCs isolated from human umbilical cord blood were identified and divided into four groups. EPCs in the Control group were cultured normally; those in the Control+HGF group were treated with HGF stimulation; those in the AD-Gab1 group were transfected with adenovirus containing the Gabl gene but not treated with HGF stimulation; and, those in the AD-Gab1+HGF group were treated with both HGF stimulation and transfection with adenovirus containing the Gabl gene. Subsequently, Gabl expression and proliferation and migration ability were compared for EPCs grown under different conditions. Furthermore, we measured phosphorylation levels of three key proteins Gab1, SHP2 and ERK1/2.

Results. The AD-Gab1+HGF group had the highest expression of Gab1 and higher proliferation and migration than the other three groups.

Conclusions. Upregulation of Gab1 promoted HGF-induced EPC proliferation and migration. Mechanistically, HGF stimulated Gab1 tyrosine phosphorylation in EPCs, thus leading to activation of extracellular regulated MAP kinase $1 / 2$, which is involved in proliferation and migration signaling.

\section{Introduction}


Heart and cardiovascular-associated conditions are major causes of death worldwide, and the number of people affected is continually increasing. According to World Health Organization statistics, 17.9 million deaths were due to cardiovascular disease in 2016 , accounting for $44 \%$ of mortality worldwide [1]. Heart valve disease makes up a large proportion of cardiovascular conditions and affects more than 100 million people worldwide [2].

Currently, mechanical or biological prostheses are the " gold standard" treatment for heart valve failure [3]. However, both types of valves cannot grow and regenerate - functions particularly important in congenital heart defects, and patients often require subsequent reoperation as they age [4]. All available prostheses have other disadvantages, such as a risk of infection, constant anticoagulation in mechanical valves, progressive degeneration in bioprostheses, and lack of a tissue source in homografts [5]. The tissue-engineering heart valve (TEHV) can potentially overcome most of the current shortcomings by providing a viable valve capable of growth, remodeling, and repair. The ultimate goal of TEHV is to produce a structure similar to the native valve structure [6]. In 2006, Cebotari et al. reported two successful clinical applications of TEHV in humans by using autologous endothelial progenitor cells (EPCs) [7]. As one of the main cell sources of TEHV, EPCs can attach, multiply, and cover the implant with an endothelial layer. However, autologous EPCs are usually insufficient in patients who require heart valve replacement, and the covering with an endothelial layer occurs slowly [8,9]. The absence of this autologous endothelial layer may cause immunological reactions. If autologous EPC proliferation and migration could be enhanced, the re-endothelization time - i.e., the time required for TEHV to form an endothelial layer - could be decreased, and the immunological reactions could be alleviated.

The Grb2-associated binder (Gab) family docking proteins are involved in the amplification and integration of signal transduction evoked by growth factors, cytokines, antigens, and numerous other molecules [10]. Gab1 has an essential role in postnatal angiogenesis and arteriogenesis via hepatocyte growth factor (HGF)/c-Met signaling. It is associated with SHP2 after stimulation with HGF, which is required for activation of ERK1/2 [11]. Downregulation of Gab1 inhibits cell proliferation and migration [12, 13]. In addition, Gab1 is required for HGF-induced EC (endothelial cell) proliferation and migration [14]. We hypothesized that Gab1 might play the same role in EPCs, which are EC precursors, and that upregulation of Gab1 in EPCs might have a positive effect on proliferation and migration.

Here, we demonstrated that upregulation of Gab1 promotes HGF-induced EPC proliferation and migration.

\section{Materials \& Methods}

\section{Isolation and culture of EPCs}

Compared with adult peripheral blood or bone marrow progenitors, cord blood progenitors have distinct proliferative advantages, and cord blood can be obtained noninvasively [15]. Human umbilical cord blood was collected from the placental cords of volunteers undergoing Cesarean section delivery. Collection occurred immediately after the delivery of the placenta to avoid clot 
80 formation. 7 placental cords were utilised in this study. The EPCs were isolated and cultured as 81 described previously $[15,16]$. Briefly, mononuclear cells were isolated from the human cord blood 82 by density gradient centrifugation over Histopaque-10771 (Sigma-Aldrich, St. Louis, MO, USA), 83 according to the manufacturer's protocol. The cells were seeded in T25 flasks pre-coated with 0.1 $84 \mathrm{mg} / \mathrm{ml}$ of human fibronectin (Sigma-Aldrich) and were incubated in EGM-2 BulletKit medium 85 (Lonza, Cologne, Germany). After 3 days, nonadherent cells were removed, and the medium was 86 replaced. Subsequently, the medium was changed every 2 days.

87

88

89

90

91

92

93

94

95

96

97

98

99

100

101

102

103

104

105

106

107

108

109

110

111

112

113

114

115

116

117

118

119

\section{Characterization of EPCs}

After 7 days of culture in vitro, the EPCs were characterized as adherent cells, which were double-positive for acetylated low-density lipoprotein (acLDL) uptake and lectin binding, as assessed by direct fluorescent staining, as described previously [17].

Briefly, to evaluate the ability of ac-LDL uptake and lectin binding in EPCs, the cells were cultured in $10 \mu \mathrm{g} / \mathrm{mL}$ of 1, 1'-dioctadecyl-3, 3, 3', 3-tetramethyl-indocarbocyanine perchloratelabeled acetylated low-density lipoprotein (Sigma-Aldrich) for 4 hours at $37^{\circ} \mathrm{C}$. They were then fixed with $2 \%$ paraformaldehyde for 15 minutes. The cells were washed with PBS and reacted with fluorescein isothiocyanate (FITC)-labeled Ulex europaeus agglutinin-1 (UEA-1, $10 \mu \mathrm{g} / \mathrm{mL}$; Sigma-Aldrich) at room temperature for 1 hour. The cells were washed to remove the free UEA1. Nuclear counterstaining was performed with DAPI (4', 6-diamidino 2-phenylindole; SigmaAldrich), and the cells were examined under a fluorescence microscope (Nikon, Tokyo, Japan). The adherent cells that stained with triple-positive fluorescence were determined to be EPCs. Nuclear staining with DAPI verified that nearly all the adherent cells $(>95 \%)$ were acLDL $(+)$ ulex-lectin $(+)$.

Expression of endothelial lineage surface markers was evaluated by flow cytometry (BD Accuri C6, BD Biosciences, USA) using PE mouse anti-human CD31 antibody (BioLegend, San Diego, USA), PE anti-human KDR antibody (BioLegend, San Diego, USA). Flow cytometry detection of hematopoietic cells was performed using antibodies against hematopoietic cell-specific surface antigen such as PE anti-human CD133 antibody (BioLegend, San Diego, USA). Appropriate isotype control was PE Human IgG1 Isotype control recombinant antibody (BioLegend, San Diego, USA). Data were analyzed by using the BD Accuri C6 software package (BD Biosciences, USA).

\section{Adenovirus transfection}

AD-Gab1 was purchased from Biowit Technologies (Shenzhen, China). EPCs were harvested with $0.25 \%$ trypsin-EDTA (Gibco, Carlsbad, CA, USA) plated and seeded on 96-well plates at a density of 8000 cells per well. After 24 hours, $100 \mu \mathrm{L}$ AD-Gab1 medium at six different multiplicity of infection (MOI) levels was added into each well for EPC transfection in triplicate. After 24 hours, the cells were examined under a fluorescence microscope, and fluorescence intensities were measured in ImageJ. A suitable MOI was determined and used for subsequent EPC transfection with AD-Gab1. After the cell intensity reached approximately $80 \%$, RNA and protein were extracted from cells. We used a PrimeScript RT Reagent Kit (Takara, Dalian, China) to synthesize cDNA, then examined the expression of Gab1 by using quantitative real-time PCR 
120

121

122

123

124

125

126

127

128

129

130

131

132

133

134

135

136

137

138

139

140

141

142

143

144

145

146

147

148

149

150

151

152

153

154

155

156

157

158

159

with SYBR Premix Ex Taq II (Takara), in triplicates run three times each. For Gab1, we used the forward primer 5'-TGCCATTAACTGTGCTTCCCA-3' and the reverse primer 5'TCGCACAGAGCACTCCAAAT-3'. For $\beta$-actin, we used the forward primer 5'CTCCATCCTGGCCTCGCTGT-3' and the reverse primer 5'-GCTGTCACCTTCACCGTTCC3'. The relative Gab1 expression was calculated in Bio-Rad CFX Manager. Protein was prepared for western blot analysis in triplicate.

\section{Measurement of cell proliferation}

EPCs that were cultured at $37^{\circ} \mathrm{C}$ and $5 \% \mathrm{CO}_{2}$ in an incubator for 14 days were harvested with $0.25 \%$ trypsin-EDTA and plated onto 96-well plates with EGM-2 (contain 5\% FBS). The cells were incubated at $37^{\circ} \mathrm{C}$ and $5 \% \mathrm{CO}_{2}$ for 24 hours. We added $0.5 \mu \mathrm{L}$ of $50 \mu \mathrm{M}$ EdU (SigmaAldrich) into each well containing $500 \mu \mathrm{L}$ of medium and incubated the cells for 4 hours. The cells were fixed with $4 \%$ paraformaldehyde and incubated with $2 \mathrm{mg} / \mathrm{mL}$ aminoacetic acid for $5 \mathrm{~min}$ with oscillation. The cells were incubated with $100 \mu \mathrm{L}$ of the penetrant into each well and oscillated $10 \mathrm{~min}$, and $100 \mu \mathrm{L}$ of $1 \times$ EdU solution was then added and incubated for 30 minutes. DAPI was used to stain cell nuclei.

After digestion with pancreatic enzyme, EPCs were plated on 96-well plates with a density of approximately 4000 cells per well, in triplicate. The next day (24 hours later), the AD-Gab1 group and $\mathrm{AD}-\mathrm{Gab} 1+\mathrm{HGF}$ group were transfected with $\mathrm{AD}-\mathrm{Gab} 1$ at $\mathrm{MOI}=20$. One plate was randomly chosen to run CCK-8 (7Sea Pharmatech, Shanghai, China) tests, to determine 24 hour proliferation. The medium of the Control+HGF group and AD-Gab1+HGF group was changed to EGM-2 with 5\% FBS and $20 \mathrm{ng} / \mathrm{mL}$ of HGF (PeproTech, Rocky Hill, NJ, USA), whereas the Control group and AD-Gab1 group were maintained in EGM-2 with 5\% FBS and without HGF. The cells were incubated for another 24 hours, and cell proliferation was then tested ( 72 hour time point). Subsequently, we removed HGF stimulation in Control+HGF group and AD-Gab1+HGF group, performed CCK-8 tests at 96 hours and 120 hours with the last two plates.

\section{In vitro wound-healing assay}

The cells were removed by trypsinization, counted, and plated at a density of $1 \times 10^{6}$ cells/per well in six-well plates. After transfection, cells were incubated until confluent monolayers were formed for wounding assays. Wounds were made with a pipette tip, and photographs were taken immediately (time 0). Then the medium of the Control+HGF group and AD-Gab1+HGF group was changed to EGM-2 without FBS and with $20 \mathrm{ng} / \mathrm{mL}$ of HGF; the Control group and AD-Gab1 group were maintained in EGM-2 without FBS. Photographs were taken at 12 hours, 24 hours, 36 hours, and 48 hours after wounding. The cell-covered area (\%) was measured to determine the amount of migration by the cell monolayer to cover the wounded area during this time period. The cell-covered area $(\%)$ was determined as $\left(\mathrm{W}_{0}-\mathrm{W}_{\mathrm{t}}\right) / \mathrm{W}_{0}$, where $\mathrm{W}_{\mathrm{t}}$ represents the wound area (with no cells) at time t. These areas were measured and analyzed in ImageJ.

\section{Western blot analysis}

Cells were harvested in RIPA bu $\square$ er containing a protease inhibitor cocktail. Total protein concentration was determined using a Bradford protein assay kit (Solarbio). Supernatant and lysis samples were separated with SDS-PAGE $(8 \%$ or $10 \%)$ and transferred onto a polyvinylidene 
160

161

162

163

164

165

166

167

168

169

170

171

172

173

174

175

176

177

178

179

180

181

182

183

184

185

186

187

188

189

190

191

192

193

194

195

196

197

198

199

difluoride membrane, blocked with 5\% non-fat dry milk and probed overnight at $4{ }^{\circ} \mathrm{C}$ with appropriate primary antibodies (Cell Signaling Technology, Danvers, MA, USA). After washing and incubation with secondary antibodies (Cell Signaling Technology, MA, USA) and visualized using Power-Opti ECLTM solution (Millipore, MA, USA) and a cooled CCD camera system (Vilber Fusion Solo 4S, PAR, France).

\section{Statistical analysis}

The original data were processed in GraphPad Prism 7.0. The quantitative real-time PCR data were analyzed with an independent-samples $t$-test. The western blot analysis in figure 1 was performed with a Student's $t$-test. Other statistical analysis was performed with ordinary one-way ANOVA. All these tests were performed on at least three independent biological replicates. All data are expressed as the mean $\pm \mathrm{SD}$. A value of $\mathrm{P}<0.05$ was considered statistically significant.

\section{Ethics approval and consent to participate}

We have received informed consent from participants. All the procedures were followed by the Medical Ethics Committee of The Affiliated Hospital of Qingdao University, Qingdao.

\section{Results}

\section{Characterization of EPCs}

Freshly isolated mononuclear cells were the small round cells suspended in the medium. With increased culturing time, the cells gradually stretched and became larger, forming cell-cell adhesions. Cell colonies were observed at approximately 7 days after seeding. Three to four weeks later, the endothelial cells showed a typical cobblestone-like appearance. After 7 days' culture in vitro, when observed under a fluorescence microscope, the adherent dil-acLDL-labeled cells were red, the cells bound with FITC-UEA-I were green, and the cell nuclei were stained blue with DAPI. Almost $100 \%$ of the cells showed three colors, and these cells were considered the differentiating EPCs (Fig. 1A, B, C, D, E, and F). The cells were positive for CD31, KDR and negative for hematopoietic cell surface antigen CD133 (Fig. 1K) which is in accordance with previously published data [18].

\section{Adenovirus transfection into EPCs}

The efficiency of adenovirus transfection was determined by fluorescence microscopy. The more EPCs transfected, the more green fluorescence was observed. An excessive MOI may damage EPCs and even lead to cell death. The statistical results indicated that, at MOI $=20$, the number of cells with green fluorescence was much greater than at other MOI levels (Fig. 1G). After we selected the proper MOI, we sought to validate the adenovirus transfection into EPCs. The qPCR results showed that the expression of the Gabl gene was upregulated in the AD-Gab1 group after transfection (Fig. 1I), and western blot analysis demonstrated that Gab1 protein expression was higher in the AD-Gabl group (Fig. 1H, J).

\section{Effect of overexpression of Gab1 on EPC proliferation}

Detection of EdU is more sensitive than BrdU detection and can be accomplished in minutes [19]. Under a fluorescence microscope, we observed EdU incorporation to determine the 
200 proliferation of EPCs that were cultured for 14 days (Fig. 2A, B, C, and D). The proliferation of 201 EPCs in the Control+HGF group and AD-Gab1 group was increased, whereas the proliferation in the AD-Gab1+HGF group was even higher, and the results were statistically significant (Fig. 2E).

In CCK-8 tests, the optical density (OD) was measured at $450 \mathrm{~nm}$ wavelength. The greater the

205

206

207

208

209

210

211

212

213

214

215

216

217

218

219

220

221

222

223

224

225

226

227

228

229

230

231

232

233

234

235

236

237

238

239 cell number, the higher the OD. Because we added HGF stimulation between 48 hours and 72 hours, the cell proliferation rate was determined as the OD value at 72 hours divided by the OD value at 48 hours. Statistical analysis of the data confirmed the results of the EdU incorporation assay. The cell proliferation ability of the Control+HGF group and AD-Gab1 group was higher than that of the Control group between 48 hours and 72 hours (Fig. 2F). The cell proliferation ability of the AD-Gab1+HGF group was significantly different from that of the other three groups (Fig. 2F). We plotted growth curves with the mean OD values as the ordinate and the cell culture time as the abscissa (Fig. 2G). After HGF stimulation, EPCs in the AD-Gab1+HGF group grew most rapidly.

In conclusion, HGF-stimulation and Gab1 have a role in promoting cell proliferation, and upregulation of Gab1 expression enhances this effect.

\section{Effect of overexpression Gab1 on EPC migration}

In wound-healing assays, we determined the area covered by a wounded cell monolayer on plastic after different treatments. When we used EGM-2 without FBS, EPCs had little proliferation ability. The results are reported as cell-covered area (fig. 3A, B). The cell-covered areas in the Control+HGF group and AD-Gab1 group were higher than that in the Control group, and the increase in cell-covered area in the Control+HGF group was higher than that in the AD-Gab1 group. The data suggested that HGF stimulation and upregulation of Gab1 both influence EPC migration. HGF stimulation had a greater effect on EPC migration than up-regulation of Gab1 expression. We presumed that HGF stimulation combined with upregulation of Gab1 to treat EPCs should result in a greater improvement in EPC migration. To test this possibility, we analyzed an AD-Gab1+HGF group. At 12 hours, the cell-covered area in the AD-Gab1+HGF group was significantly higher than those in the other three groups (Fig. 3A). At 24 hours, 36 hours, and 48 hours, the cell-covered area in the AD-Gab1+HGF group was higher than those in the other groups (Fig. 3A) and increased more quickly than those in the other groups (Fig. 3C). Thus, EPCs treated with both HGF stimulation and transfection of adenovirus containing the Gab1 gene had a greater migration ability.

\section{HGF-mediated signaling in EPCs}

The ERK signaling pathway is involved in cell proliferation and migration. To investigate how upregulation of Gab1 mediates HGF-induced EPC proliferation and migration, we assessed the activation of ERK1/2 in response to HGF stimulation and upregulation of Gab1 expression. The expressions of c-Met, Gab1, phospho-Gab1, SHP2, phospho-SHP2, ERK1/2, and phosphoERK1/2 in EPCs was analyzed via western blotting. The expression of the c-Met in ADGab1+HGF group was consistent with the other groups (Fig. 4E). Quantitative analysis demonstrated that the phosphorylation of Gab1 in the AD-Gab1 group was almost equal to that in the Control group but lower than that in the Control+HGF group and AD-Gab1+HGF group (Fig. 
240

241

242

243

244

245

246

247

248

249

250

251

252

253

254

255

256

257

258

259

260

261

262

263

264

265

266

267

268

269

270

271

272

273

274

275

276

277

278

279

4B). We confirmed that HGF stimulated Gab1 tyrosine phosphorylation, as previously reported [14]. Once sufficient Gab1 expression was achieved, for example in the AD-Gab1+HGF group, HGF induced more phosphorylation of Gab1 (Fig. 4B). The increased Gab1 phosphorylation substantially increased the levels of SHP2 phosphorylation, thus leading to activation of MAP kinase $1 / 2$, which is involved in proliferation and migration signaling (Fig. 4C, D). Together, these results established a critical role of EPCs Gab1 in proliferation and migration signaling and indicated that upregulation of Gab1 enhances the function of this signaling pathway.

\section{Discussion}

Asahara et al. first reported that a subtype of hematopoietic progenitor cells from adults, namely EPCs [20], have EC (endothelial cell) features and can differentiate into mature ECs and might represent a potential strategy for TEHV. Subsequently, experiments confirmed that TEHV using autologous EPCs is very encouraging [7, 21, 22]. However, EPCs are a fairly rare cell population, and, when administered intravenously, only a very small fraction of EPCs reach the target region and participate in re-endothealization. How to increase their cell number is an urgent problem.

Many Receptor tyrosine kinases (RTKs), including vascular endothelial growth factor (VEGF), are shown to regulate EC function [23]. Except VEGF, hepatocyte growth factor (HGF) is a angiogenic factor which stimulates EC proliferation and migration [24, 25]. HGF binds its receptor c-Met and stimulates c-Met kinase activation, which triggers transphosphorylation of c-Met and downstream signaling events [26]. The $\mathrm{HGF} / \mathrm{c}-$ Met pathway has emerged as a promising therapeutic target by which to promote re-endothealization in vivo.

Unlike most RTKs recruit Gab1 indirectly via Grb2, Gab1 can be recruited to activated c-Met through direct mechanism, interacts with tyrosine-phosphorylated c-Met via the Met-binding domain (MBD, amino acids 450-532) [27, 28]. Mechanistically, we found that Gab1 is critical in mediating $\mathrm{HGF} / \mathrm{c}-\mathrm{Met}$ proliferation and migration signaling of human EPCs in vitro. To our knowledge, this is the first study revealing a key role of upregulation of Gab1 in promoting proliferation and migration and HGF-mediated signaling in human EPCs.

Previous studies on the role of Gab1 have usually used a Gab1-ecKO mice model. Those studies have found that Gab1-deficient mice have developmental defects in postnatal angiogenesis [13, 29]. However, the effect of upregulating Gab1 in human EPCs has not been explored. Using human umbilical cord blood, we isolated human EPCs and upregulated their Gab1 expression through adenovirus transfection. We showed that upregulation of Gab1 markedly enhanced the proliferation and migration of human EPCs. HGF have been shown to mobilize and increase EPC number [30]. Our results showed that under the same dose of HGF stimulation, the proliferation and migration of human EPCs was strongly enhanced in EPCs in which Gab1 was upregulated. Therefore, upregulation of Gab1 amplifies stimulation by HGF.

The results also supported that Gab1 is important for HGF-induced ERK1/2 phosphorylation. Few studies in other cells, such as HUVEC and MDCK cells, have evaluated the role of Gab1 in HGF-induced ERK1/2 [31-33]. In these cells, which were transfected to express mutated Gab1 
280

281

282

283

284

285

286

287

288

289

290

291

292

293

294

295

296

297

298

299

300

301

302

303

304

305

306

307

308

309

310

311

312

313

314

315

316

317

318

319

320

321

322

unable to recruit SHP2, the HGF induced sustained activation of the ERK pathway was found to be reduced [31]. ERK1/2 activation has been shown to regulate cell migration and survival signaling pathways [34]. Gab1 plays an important role in mediating growth factor-induced activation of ERK1/2 through recruiting SHP2 in a tyrosine phosphorylation-dependent manner [33]. However, the role of Gab1 in HGF mediated signaling in human EPCs remains unclear. Our results suggested that upregulation of Gab1 in human EPCs results in recruitment of more SHP2. We further found that under the same dose of HGF stimulation, greater SHP2 tyrosine phosphatase activation and ERK1/2 phosphorylation were observed in cells overexpressing Gab1. The data suggest signaling mechanisms by which Gab1 mediates growth factor-induced proliferation and migration.

\section{Conclusions}

In this study, we found that upregulation of Gab1 promotes HGF-induced EPC proliferation and migration. Mechanistically, HGF stimulates Gab1 tyrosine phosphorylation in EPCs, thus leading to activation of extracellular regulated MAP kinase $1 / 2$, which is involved in proliferation and migration signaling. Our findings may have clinical implications: they suggest that enhancing Gab1 signaling may be a potential strategy to increase EPC cell number and provide a new means of achieving rapid TEHV re-endothealization.

\section{Acknowledgements}

We wish to thank all colleagues at the Institute for Translation Medicine of Qingdao University for help during all phases of the project.

\section{References}

1. WHO. World Health Statistics 2018: Monitoring health for the SDGs. 2018; Available from: http://www.who.int/gho/publications/world health statistics/2018/en/.

2. Members, W.G., A.S. Go, D. Mozaffarian, V.L. Roger, E.J. Benjamin, J.D. Berry, M.J. Blaha, S. Dai, E.S. Ford, and C.S. Fox, Heart Disease and Stroke Statistics-2014 Update: A Report From the American Heart Association. Circulation, 2015. 129(4): p. e28e292.

3. Baumgartner, H., V. Falk, J.J. Bax, M. De Bonis, C. Hamm, P.J. Holm, B. lung, P. Lancellotti, E. Lansac, D. Rodriguez Munoz, R. Rosenhek, J. Sjogren, P. Tornos Mas, A. Vahanian, T. Walther, O. Wendler, S. Windecker, J.L. Zamorano, and E.S.C.S.D. Group, 2017 ESC/EACTS Guidelines for the management of valvular heart disease. Eur Heart J, 2017. 38(36): p. 2739-2791.

4. Blum, K.M., J.D. Drews, and C.K. Breuer, Tissue-Engineered Heart Valves: A Call for Mechanistic Studies. Tissue Eng Part B Rev, 2018.

5. Best, C., E. Onwuka, V. Pepper, M. Sams, J. Breuer, and C. Breuer, Cardiovascular Tissue Engineering: Preclinical Validation to Bedside Application. Physiology, 2016. 31(1): p. 7. 
323

324

325

326

327

328

329

330

331

332

333

334

335

336

337

338

339

340

341

342

343

344

345

346

347

348

349

350

351

352

353

354

355

356

357

358

359

360

361

362

363

364

365

366

367

368

369

370

371

372

373

6. Emmert, M.Y., B. Weber, L. Behr, S. Sammut, T. Frauenfelder, P. Wolint, J. Scherman, D. Bettex, J. Grunenfelder, V. Falk, and S.P. Hoerstrup, Transcatheter aortic valve implantation using anatomically oriented, marrow stromal cell-based, stented, tissueengineered heart valves: technical considerations and implications for translational cellbased heart valve concepts. Eur J Cardiothorac Surg, 2014. 45(1): p. 61-8.

7. Cebotari, S., A. Lichtenberg, I. Tudorache, A. Hilfiker, H. Mertsching, R. Leyh, T. Breymann, K. Kallenbach, L. Maniuc, A. Batrinac, O. Repin, O. Maliga, A. Ciubotaru, and A. Haverich, Clinical application of tissue engineered human heart valves using autologous progenitor cells. Circulation, 2006. 114(1 Suppl): p. I132-7.

8. Rippel, R.A., H. Ghanbari, and A.M. Seifalian, Tissue-engineered heart valve: future of cardiac surgery. World J Surg, 2012. 36(7): p. 1581-91.

9. Bianconi, V., A. Sahebkar, P. Kovanen, F. Bagaglia, B. Ricciuti, P. Calabro, G. Patti, and M. Pirro, Endothelial and cardiac progenitor cells for cardiovascular repair: A controversial paradigm in cell therapy. Pharmacol Ther, 2018. 181: p. 156-168.

10. Nakaoka, Y. and I. Komuro, Gab docking proteins in cardiovascular disease, cancer, and inflammation. Int J Inflam, 2013. 2013: p. 141068.

11. Aasrum, M., J. Odegard, D. Sandnes, and T. Christoffersen, The involvement of the docking protein Gab1 in mitogenic signalling induced by EGF and HGF in rat hepatocytes. Biochim Biophys Acta, 2013. 1833(12): p. 3286-94.

12. Sang, H., T. Li, H. Li, and J. Liu, Down-regulation of Gab1 inhibits cell proliferation and migration in hilar cholangiocarcinoma. PLoS One, 2013. 8(11): p. e81347.

13. Xu, L., J. Li, Z. Kuang, Y. Kuang, and H. Wu, Knockdown of Gab1 Inhibits Cellular Proliferation, Migration, and Invasion in Human Oral Squamous Carcinoma Cells. Oncol Res, 2017.

14. Zhao, J., W. Wang, C.H. Ha, J.Y. Kim, C. Wong, E.M. Redmond, A. Hamik, M.K. Jain, G.S. Feng, and Z.G. Jin, Endothelial Grb2-associated binder 1 is crucial for postnatal angiogenesis. Arterioscler Thromb Vasc Biol, 2011. 31(5): p. 1016-23.

15. Murohara, T., H. Ikeda, J. Duan, S. Shintani, K. Sasaki, H. Eguchi, I. Onitsuka, K. Matsui, and T. Imaizumi, Transplanted cord blood-derived endothelial precursor cells augment postnatal neovascularization. J Clin Invest, 2000. 105(11): p. 1527-36.

16. Li, X., C. Chen, L. Wei, Q. Li, X. Niu, Y. Xu, Y. Wang, and J. Zhao, Exosomes derived from endothelial progenitor cells attenuate vascular repair and accelerate reendothelialization by enhancing endothelial function. Cytotherapy, 2016. 18(2): p. 25362.

17. Roberts, N., Q. Xiao, G. Weir, Q. Xu, and M. Jahangiri, Endothelial progenitor cells are mobilized after cardiac surgery. Ann Thorac Surg, 2007. 83(2): p. 598-605.

18. Ingram, D.A., L.E. Mead, H. Tanaka, V. Meade, A. Fenoglio, K. Mortell, K. Pollok, M.J. Ferkowicz, D. Gilley, and M.C. Yoder, Identification of a novel hierarchy of endothelial progenitor cells using human peripheral and umbilical cord blood. Blood, 2004. 104(9): p. 2752-60.

19. Salic, A. and T. Mitchison, A chemical method for fast and sensitive detection of DNA synthesis in vivo. Proc. Natl. Acad. Sci. U.S.A., 2008. 105(7): p. 2415-20.

20. Asahara, T., T. Murohara, A. Sullivan, M. Silver, R. van der Zee, T. Li, B. Witzenbichler, G. Schatteman, and J.M. Isner, Isolation of putative progenitor endothelial cells for angiogenesis. Science, 1997. 275(5302): p. 964-7.

21. Dohmen, P.M., A. Lembcke, S. Holinski, D. Kivelitz, J.P. Braun, A. Pruss, and W. Konertz, Mid-Term Clinical Results Using a Tissue-Engineered Pulmonary Valve to Reconstruct the Right Ventricular Outflow Tract During the Ross Procedure. The Annals of Thoracic Surgery, 2007. 84(3): p. 729-736.

22. Sales, V.L., B.A. Mettler, G.C. Engelmayr, E. Aikawa, J. Bischoff, D.P. Martin, A. Exarhopoulos, M.A. Moses, F.J. Schoen, M.S. Sacks, and J.E. Mayer, Endothelial 
374

375

376

377

378

379

380

381

382

383

384

385

386

387

388

389

390

391

392

393

394

395

396

397

398

399

400

401

402

403

404

405

406

407

408

409

410

411

412

413

414

415

416

Progenitor Cells as a Sole Source for Ex Vivo Seeding of Tissue-Engineered Heart Valves. Tissue Engineering Part A, 2010. 16(1): p. 257-267.

23. Anne, L., K. Thomas, N. L Christian, S. Wolfgang, D. Helmut, and F.P. Limbourg, Evaluation of postnatal arteriogenesis and angiogenesis in a mouse model of hind-limb ischemia. Nature Protocols, 2009. 4(12): p. 1737-1746.

24. Eric, A., Y. Jun, Z. Stefan, I. Yasuko, S. Carsten, S. Rei, O. Noriyuki, R.M. Easton, G. Gennaro, and M.J. Birnbaum, Akt1/protein kinase Balpha is critical for ischemic and VEGF-mediated angiogenesis. Journal of Clinical Investigation, 2005. 115(8): p. 21192127.

25. Angelika, B., C. Guillaume, I. Masayoshi, M. Marina, K. Masamichi, F. Ariane, B. Jana, F. Henrik, D. Carmen, and O. Kisho, MicroRNA-92a controls angiogenesis and functional recovery of ischemic tissues in mice. Science, 2009. 324(5935): p. 1710-1713.

26. Hoon, H.C., W. Weiye, J. Bong Sook, W. Chelsea, H. Angelika, P. Klaus, T.A. Mckinsey, E.N. Olson, and J. Zheng-Gen, Protein kinase D-dependent phosphorylation and nuclear export of histone deacetylase 5 mediates vascular endothelial growth factor-induced gene expression and angiogenesis. Journal of Biological Chemistry, 2008. 283(21): p. 145909.

27. Gu, H. and B.G. Neel, The 'Gab' in signal transduction. Trends in Cell Biology, 2003. 13(3): p. 122-130.

28. Barrow-McGee, R. and S. Kermorgant, Met endosomal signalling: In the right place, at the right time. The International Journal of Biochemistry \& Cell Biology, 2014. 49: p. 69-74.

29. Wang, W., S. Xu, M. Yin, and Z.G. Jin, Essential roles of Gab1 tyrosine phosphorylation in growth factor-mediated signaling and angiogenesis. Int J Cardiol, 2015. 181: p. 180-4.

30. Rehman, J., J. Li, L. Parvathaneni, G. Karlsson, V.R. Panchal, C.J. Temm, J. Mahenthiran, and K.L. March, Exercise acutely increases circulating endothelial progenitor cells and monocyte-/macrophage-derived angiogenic cells is. Journal of the American College of Cardiology, 2004. 43(12): p. 2314.

31. Maroun, C., M. Naujokas, M. Holgado-Madruga, A. Wong, and M. Park, The tyrosine phosphatase SHP-2 is required for sustained activation of extracellular signal-regulated kinase and epithelial morphogenesis downstream from the met receptor tyrosine kinase. Mol. Cell. Biol., 2000. 20(22): p. 8513-25.

32. Shioyama, W., Y. Nakaoka, K. Higuchi, T. Minami, Y. Taniyama, K. Nishida, H. Kidoya, T. Sonobe, H. Naito, Y. Arita, T. Hashimoto, T. Kuroda, Y. Fujio, M. Shirai, N. Takakura, R. Morishita, K. Yamauchi-Takihara, T. Kodama, T. Hirano, N. Mochizuki, and I. Komuro, Docking protein Gab1 is an essential component of postnatal angiogenesis after ischemia via HGF/c-met signaling. Circ. Res., 2011. 108(6): p. 664-75.

33. Aasrum, M., J. Odegard, G.H. Thoresen, I.J. Brusevold, D.L. Sandnes, and T. Christoffersen, Gab1 amplifies signaling in response to low-intensity stimulation by HGF. Cell Biol Int, 2015. 39(10): p. 1177-84.

34. Koch, S., S. Tugues, X. Li, L. Gualandi, and L. Claessonwelsh, Signal transduction by vascular endothelial growth factor receptors. Cold Spring Harbor Perspectives in Medicine, 2011. 2(7): p. a006502. 


\section{Figure 1}

\section{Characterization of EPCs}

Fig. 1 Immunofluorescence staining of EPCs was pe $r$ formed after 7 days (orig. mag. $\times 40$ ). (A) Unstained EPCs; (B) d il-acLDL-labeled cells (red); (C) FITC-UEA-I-bound cells (green); (D) DAPI contrast staining of the cell nucle i ; (E) d il-acLDL-labeled and FITC-UEA-I-bound double positive cells; (F) d il-acLDL-labeled, FITCUEA-I-bound, and DAPI stained triple positive cells. Fluorescence indicating Gabl expression in EPCS transfected with adenovirus (24 hours) with different multiplicity of infection (MOI) levels, and expression of Gab1 at the optimal MOI. (G) The number of fluorescent cells at different MOI levels. (H) qPCR results show the expression of the Gab1. (I, J) The expression of the Gabl after transfected (western blot). (K) Flow cytometry characterization of EPCs for CD31, KDR and CD133. Plots depict control isotype IgG staining (black histograms) versus specific antibody staining (empty histograms). ${ }^{* *} \mathrm{p}<0.01$ ( $\mathrm{n}=3$ ) versus other MOl groups, ${ }^{*} p<0.05(n=3)$ versus Control, ${ }^{* * *} p<0.001(n=3)$ versus Control. Values are the mean \pm SD. 

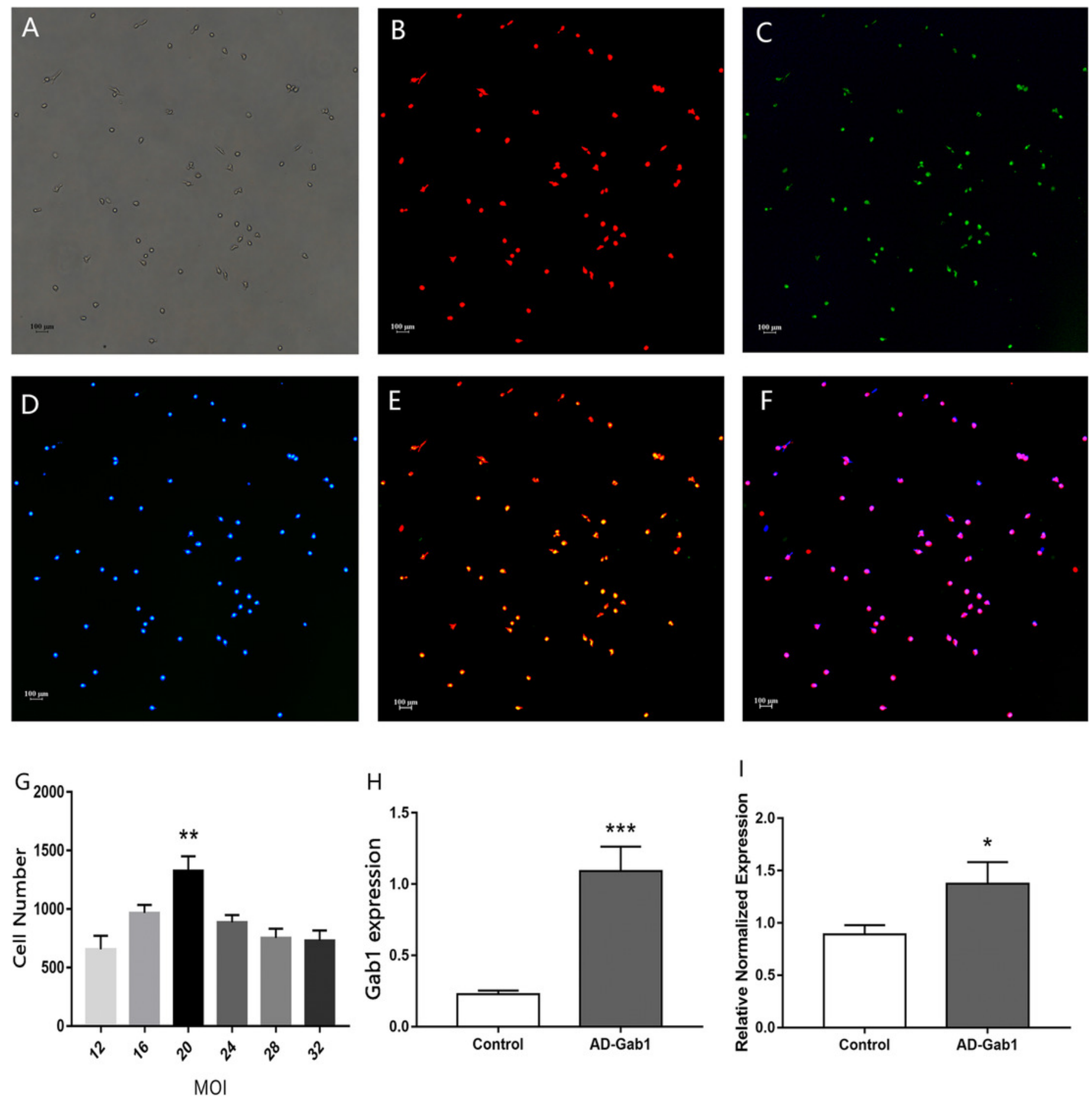

J
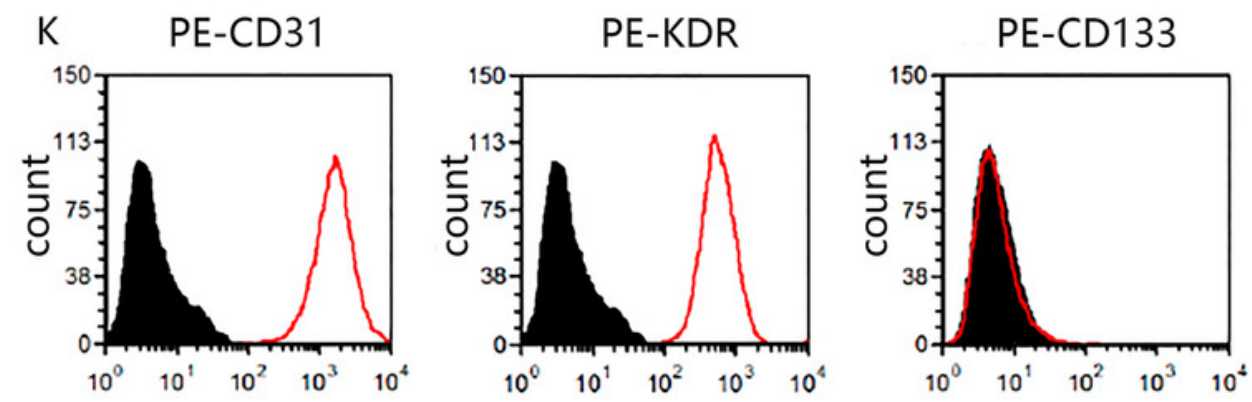


\section{Figure 2}

Ed U incorporation reflec ting the proliferationof EPCS

Fig. $2 \mathrm{Ed} \mathrm{U} \mathrm{incorporation} \mathrm{reflec} \mathrm{ting} \mathrm{the} \mathrm{proliferation} \mathrm{of} \mathrm{EPCs} \mathrm{cultured} \mathrm{for} 14$ days. (A), (B), (C), (D) DAPI (blue) was used to stain nucle i, and Ed U (green) was incorporated into EPCs in each group (orig. mag. $\times 100$ ). (E) The ratio of EPCs stained by Ed $U$ in each group. $* p<0.05$ $(n=5)$ versus Control+HGF and AD-Gab1 group, ${ }^{* *} p<0.01(n=5)$ versus Control, Control+HGF and AD-Gab1 group. CCK-8 cell viability tests reflect ing the proliferation of EPCs in different groups. (F) Cell proliferation in each group. (G) Growth curves of EPCs in different time and treatment conditions. ${ }^{* 1} p<0.05(n=4)$ versus $C$ ontrol, C ontrol+HGF and AD-Gab1 group. ${ }^{* 2} p<0.05(n=4)$ versus $C$ ontrol+HGF and AD-Gab1 group. Values are the mean \pm SD. 


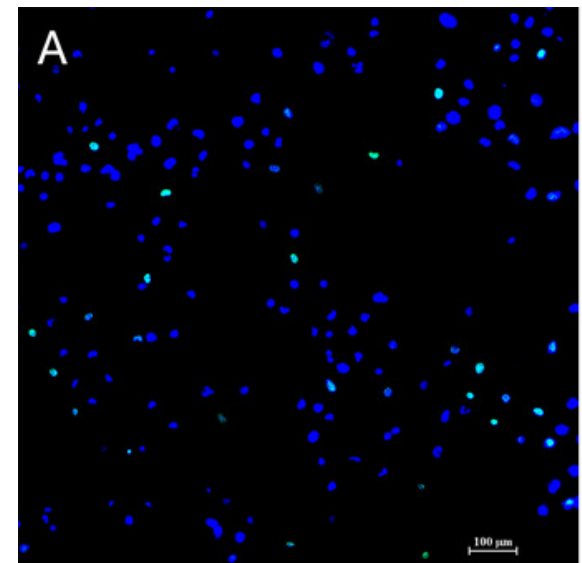

Control
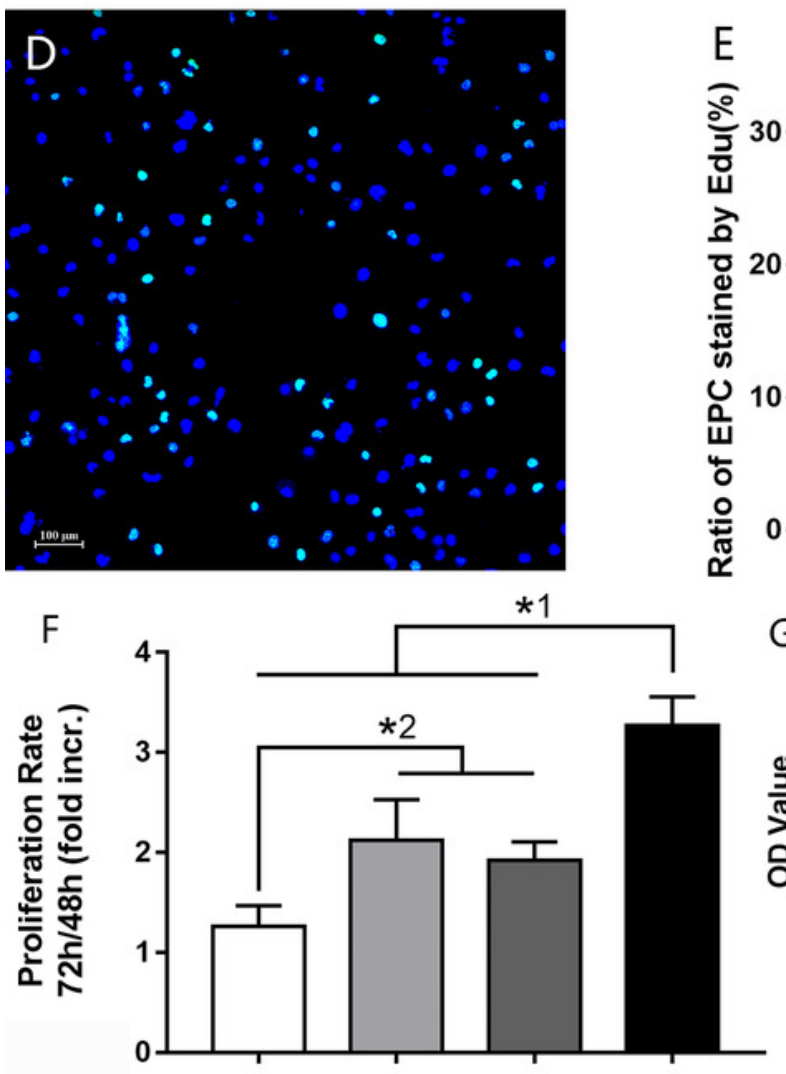

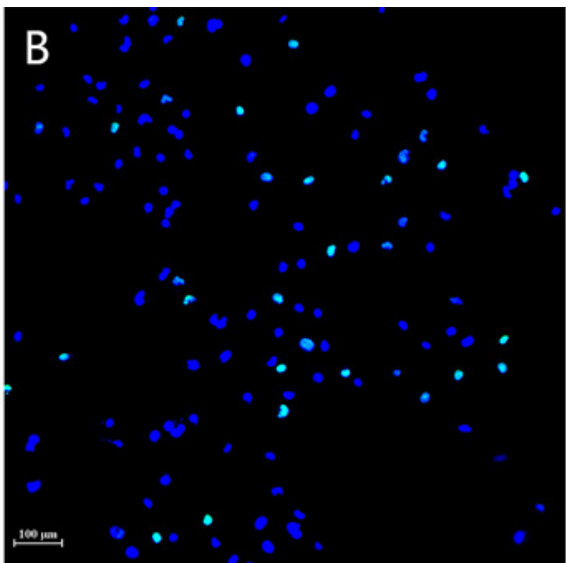

Control+HGF
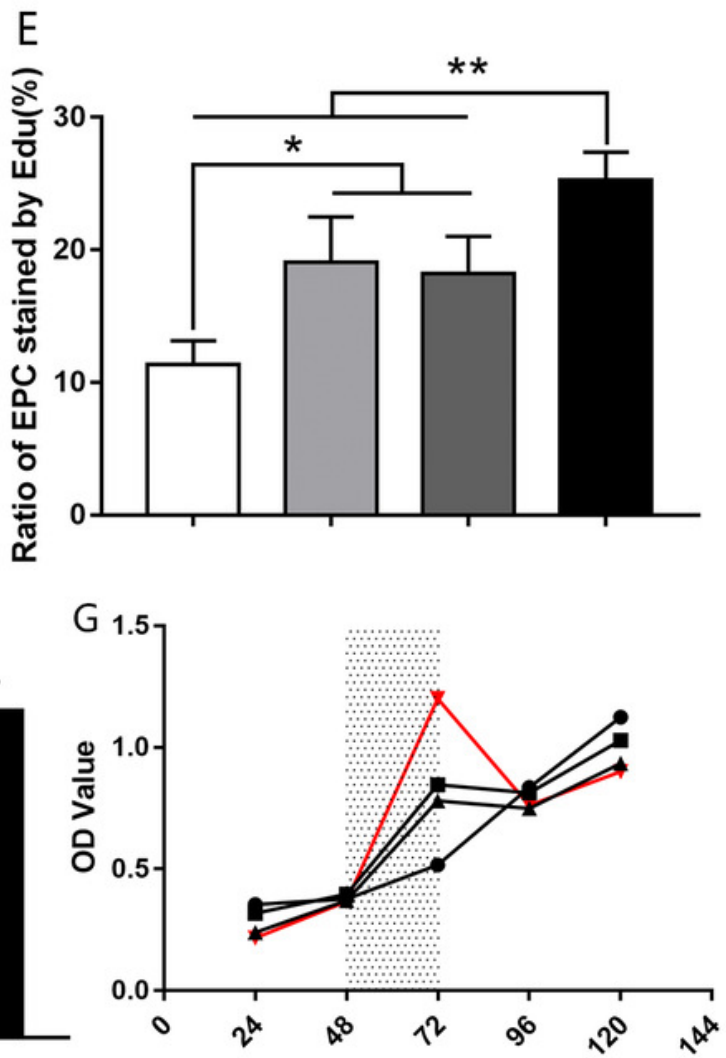

Time/h

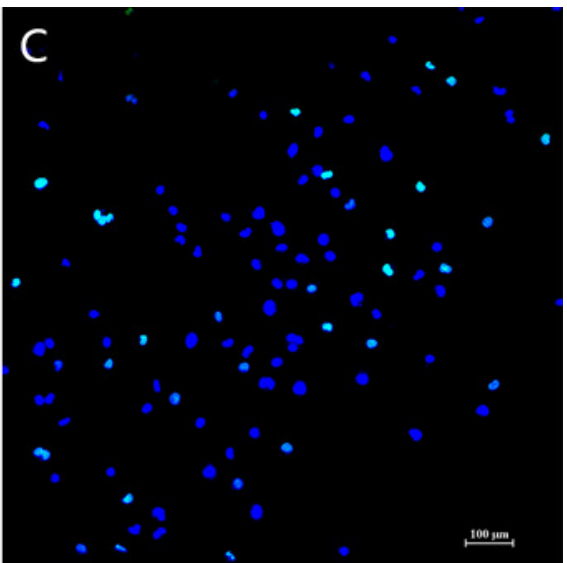

AD-Gab1
Control

$\square$ Control+HGF

$\square$ AD-Gab1

AD-Gab1+HGF $\rightarrow$ Control

- Control+HGF

$\mp$ AD-Gab1

$\rightarrow$ AD-Gab1+HGF

Add HGF stimulation 
Figure 3

Wound-healing assays reflect ing the migration of EPCs in different groups

Fig. 3 (A) Wound-healing assays reflect ing the migration of EPCs in different groups. (B) Cell-covered area in each group at different time $S$. (C) Cell-covered area increased over time. $* p<0.05(n=3)$ versus $C$ ontrol, Control+HGF and AD-Gabl group. Values are the mean \pm SD.

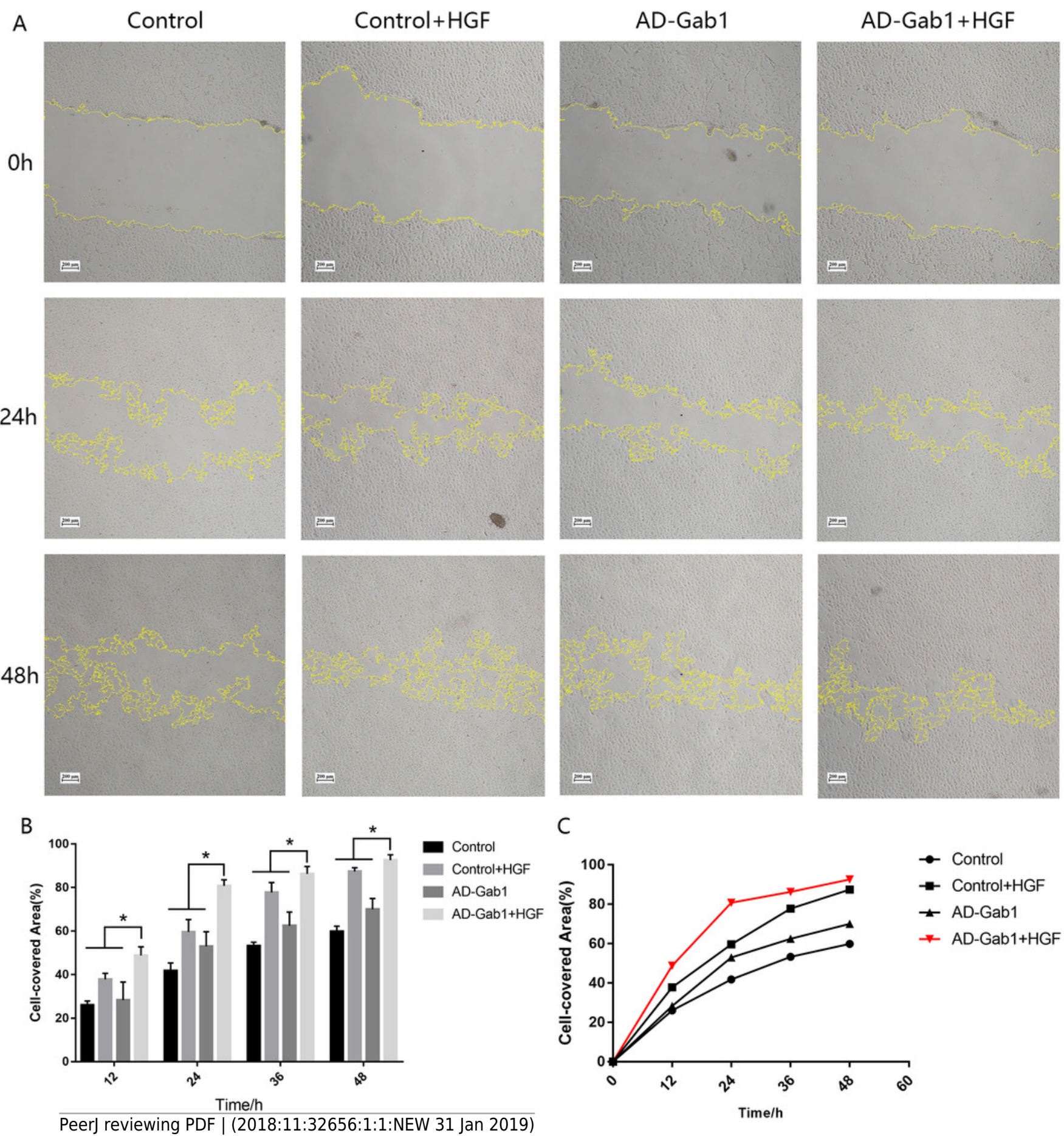




\section{Figure 4}

Effect of overexpression of Gab1 on HGF-induced phosphorylation of SHP2 and ERK1/2

Fig. 4 Effect of overexpression of Gab1 on HGF-induced phosphorylation of SHP2 and ERK1/2. The expression of c-Met, the phosphorylation of SHP2 and ERK1/2 was determined by western blot. Representative blots (A) and bar graphs summarizing the expression of c-Met (E) and the effects of the overexpression of the Gab1 (B) on the HGF-induced ( $20 \mathrm{ng} / \mathrm{mL}$ ) phosphorylation of SHP2 (C) and ERK1/2 (D). *P $<0.05(n=3)$ vs C ontrol, C ontrol+HGF and AD-Gab1 group. Values are the mean \pm SD.

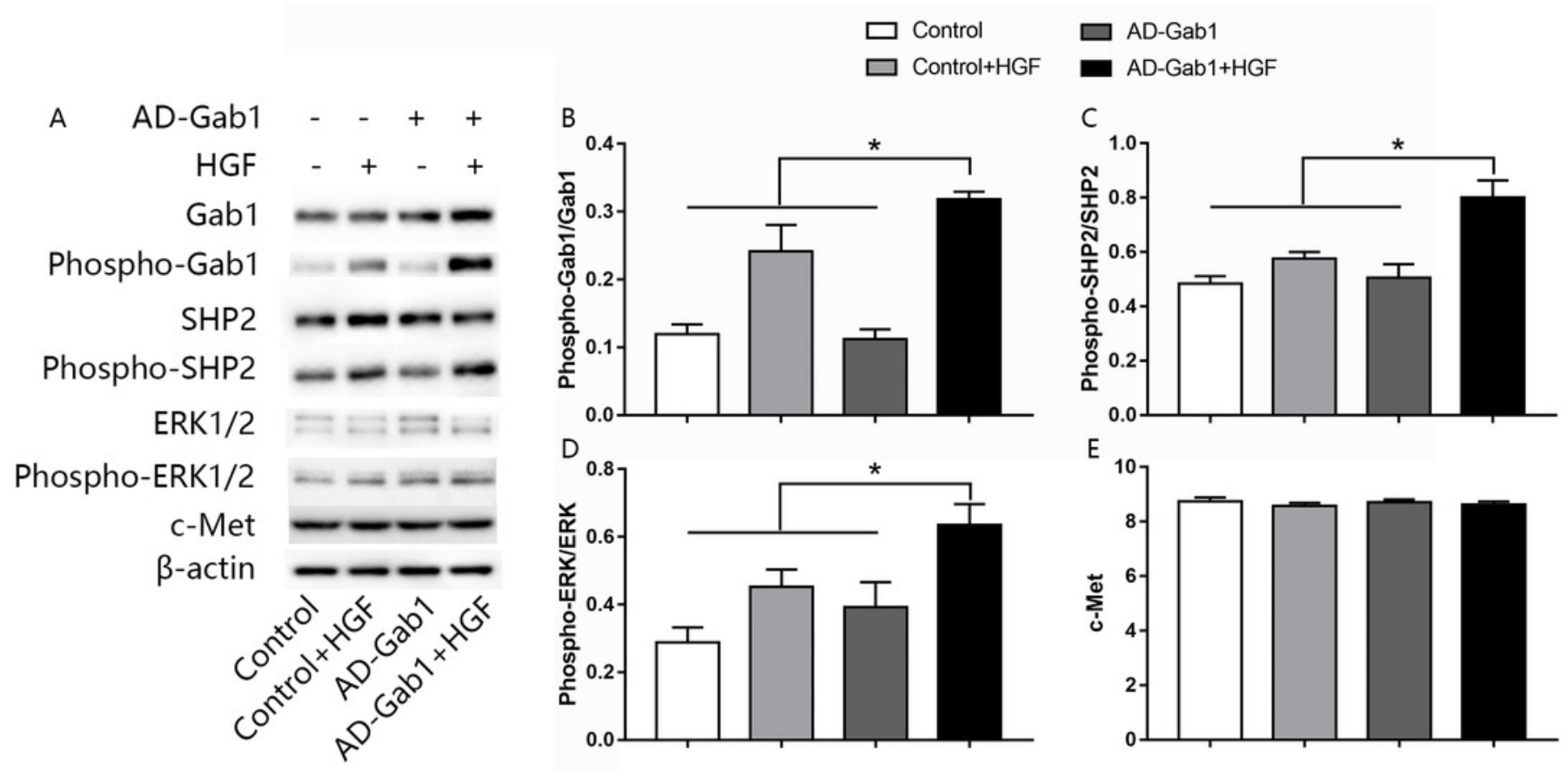

